\title{
CARCINOMA DE PALADAR BLANDO. DESCRIPCIÓN DE UNA SERIE DE CASOS
}

\section{Soft palate carcinoma. Description of cases}

\author{
Marta ZABALETA-LÓPEZ; Laia RAMOS-CASADEMONT; Estrella GÓMEZ-TOMÉ; \\ Pedro DÍAZ DE CERIO-CANDUELA; Cristina IBÁÑEZ-MUÑOZ; María Inmaculada MARTÍNEZ-TORRE \\ Hospital San Pedro. Servicio de Otorrinolaringología y Cirugía Cérvico-Facial. Logroño. España. \\ Correspondencia: mzabaletalopez@gmail.com
}

Fecha de recepción: 1 de julio de 2020

Fecha de aceptación: 13 de julio de 2020

Fecha de publicación: 15 de julio 2020

Fecha de publicación del fascículo: 1 de diciembre de 2020

Conflicto de intereses: Los autores declaran no tener conflictos de intereses

Imágenes: Los autores declaran haber obtenido las imágenes con el permiso de los pacientes

Política de derechos y autoarchivo: se permite el autoarchivo de la versión post-print (SHERPA/RoMEO)

Licencia CC BY-NC-ND. Licencia Creative Commons Atribución-NoComercial-SinDerivar 4.0 Internacional

Universidad de Salamanca. Su comercialización está sujeta al permiso del editor

RESUMEN: Introducción y objetivo: Describir las características clínicas, anatómicas y el tratamiento quirúrgico, incluyendo las distintas opciones de reconstrucción de los tumores de paladar blando. Descripción: Estudio retrospectivo de 6 pacientes diagnosticados y tratados en el servicio de Otorrinolaringología del hospital San Pedro de Logroño entre los años 2018 y 2019. Discusión: Exponemos los resultados de nuestros pacientes y realizamos una revisión de los tumores de orofaringe localizados en el paladar blando, los detalles anatómicos y técnicas quirúrgicas reconstructivas más utilizadas en el tratamiento quirúrgico de los tumores de paladar blando. Conclusiones: La prevalencia de los tumores de orofaringe ha aumentado en los últimos años a expensas de la infección por el virus del papiloma humano (VPH). Sin embargo, los carcinomas de paladar blando son mayoritariamente P16 negativos. Estos tumores son poco sintomáticos en fases iniciales y es necesario un alto índice de sospecha para evitar diagnósticos tardíos. El desarrollo de la cirugía transoral ha impulsado el tratamiento quirúrgico de los tumores de paladar blando y puede reducir la necesidad de tratamientos complementarios hasta en un 40\% de los casos. La reconstrucción se hace necesaria en resecciones mayores del $50 \%$.

PALABRAS CLAVE: Anatomía; carcinoma; orofaringe; paladar blando; procedimientos quirúrgicos reconstructivos. 


\title{
CARCINOMA DE PALADAR BLANDO. DESCRIPCIÓN DE UNA SERIE DE CASOS \\ ZABALETA-LÓPEZ M; RAMOS-CASADEMONT L; GÓMEZ-TOMÉ E; DÍAZ DE CERIO-CANDUELA P; IBÁÑEZ-MUÑOZ C; MARTÍNEZ-TORRE M I
}

\begin{abstract}
SUMMARY: Introduction and objective: To describe clinical and anatomical features, and surgical treatment including several techniques of reconstructive surgery of soft palate carcinomas. Description: We conducted a retrospective study with 5 patients. All of them were diagnosed and treated in our department during 2018 and 2019. Discussion: We present our results and make a review on soft palate tumors pointing out anatomical details and most employed reconstructive surgical techniques for soft palate carcinoma's removal. Conclusions: The incidence of oropharyngeal carcinoma is increasing world-wide essentially due to increasing prevalence of human papillomavirus. Soft palate carcinomas are typically HPV negative. Initially, soft palate tumors are silent, and we should suspect them to avoid late diagnosis. Transoral surgery development has promoted surgical treatment in these tumors and allows better management and need for adjuvant therapy. Reconstruction is mandatory when resection is over $50 \%$ of the soft palate.
\end{abstract}

KEYWORDS: Anatomy; carcinoma; oropharynx; soft palate; surgical reconstructive procedures.

\section{INTRODUCCIÓN}

Los carcinomas del paladar blando representan el 5-15\% de los tumores de orofaringe [1]. La incidencia de estos últimos ha aumentado en los últimos años a expensas de la infección por el virus del papiloma humano (VPH) [2].

Sin embargo, los carcinomas de paladar blando son mayoritariamente P16 negativos [3]. El diagnóstico de los tumores suele ser tardío, ya que, en fases iniciales, son asintomáticos [4]. El tratamiento de elección en los tumores de paladar blando durante años ha sido la radioterapia sola o combinada a quimioterapia [5]. El empleo del abordaje transoral en este tipo de tumores y su menor morbilidad, han promovido el tratamiento quirúrgico. Una resección del paladar blando mayor del $50 \%$ en los carcinomas de esta localización exige una reconstrucción minuciosa para conseguir un buen resultado funcional [6].

\section{DESCRIPCIÓN}

Durante los años 2018 y 2019 diagnosticamos y tratamos en nuestro servicio 6 pacientes con carcinoma de paladar blando.

Caso 1. Mujer de 90 años con antecedentes de mastectomía más quimioterapia por neoplasia de mama en 2002 y carcinoma escamoso de cuero cabelludo intervenido en 2010. Se diagnosticó de carcinoma escamoso infiltrante bien diferenciado localmente avanzado de paladar duro y blando (T4aN0M0) por el servicio de Odontoestomatología. Tras valorar el caso en el comité de tumores, se realizó radioterapia tridimensional sobre tumor en paladar con intención paliativa. Se administró dosis de $20 \mathrm{~Gy}$ en 5 fracciones de $4 \mathrm{~Gy}$ cada una de ellas, con buena tolerancia. En la tomografía computarizada (TC) de control al año, no se vió tumor en cavidad oral ni extensión cervical ni pulmonar. En seguimiento en consultas externas, ha disminuido mucho el tamaño del tumor y la paciente se encuentra estable.

Caso 2. Paciente de 71 años que presentó carcinoma escamoso ulcerado, focalmente queratinizante y moderadamente diferenciado (G2) de paladar blando pT2pN1Mx (Figura 1) que se trató mediante exéresis con reconstrucción con colgajo miomucoso de arteria facial (FAMM) y bola de Bichat y vaciamiento ganglionar bilateral en septiembre de 2018. El paciente no ha seguido revisiones por traslado a otra comunidad.

CAso 3. Paciente de 64 años fumadora activa, con carcinoma escamoso de maxilar superior localmente avanzado, con nódulos pulmonares milimétricos (sospechosos de metástasis, pero no confirmados histopatológicamente) T4aN1M1. Tratada con 5 ciclos de cisplatino y 5 -Fluorouracilo con cetuximab y posteriormente, radioterapia más quimioterapia con cisplatino (6 ciclos), finalizando 


\section{CARCINOMA DE PALADAR BLANDO. DESCRIPCIÓN DE UNA SERIE DE CASOS \\ ZABALETA-LÓPEZ M; RAMOS-CASADEMONT L; GÓMEZ-TOMÉ E; DÍAZ DE CERIO-CANDUELA P; IBÁÑEZ-MUÑOZ C; MARTÍNEZ-TORRE M I}

la radioterapia en noviembre 2018. En la última TC se apreciaron cambios postratamiento con remisión local completa y estabilidad de lesiones pulmonares. Falleció a los 26 meses por infección respiratoria.

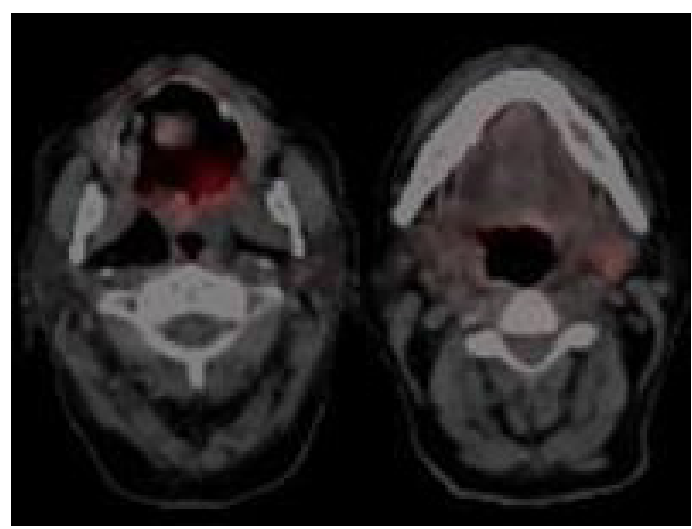

Figura 1. PET de carcinoma paladar blando con imagen del tumor primario y de adenpatía metastásica.

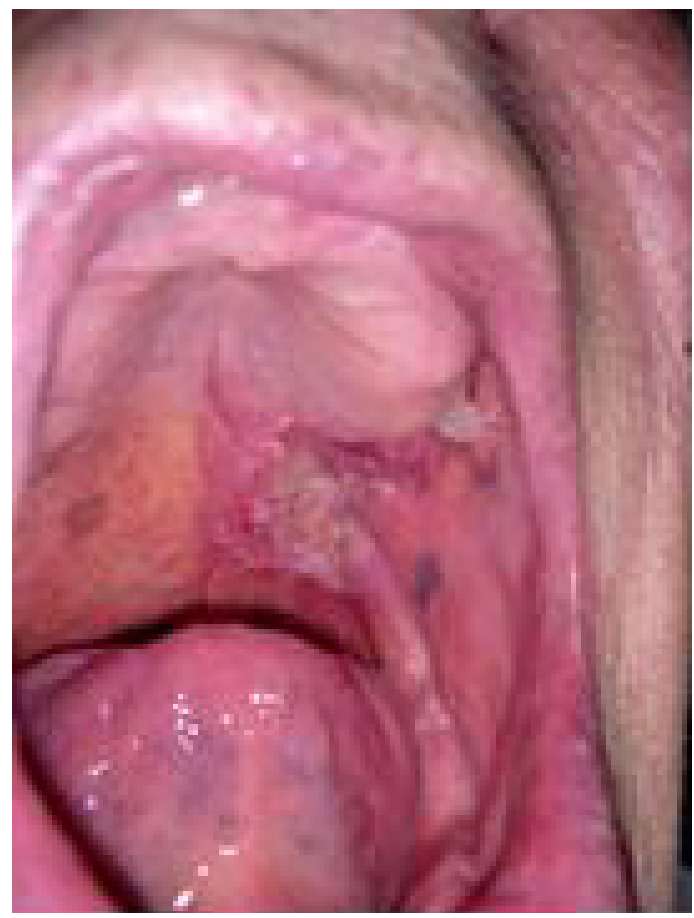

Figura 2 Carcinoma paladar blando.
CAso 4. Paciente de 64 años diagnosticado de carcinoma adenoide quístico pT3 pN0 M0 (Figura 2) tratado con maxilectomía y reconstrucción con colgajo temporal, colocación de prótesis de temporal y vaciamiento ganglionar en noviembre de 2019. Tres semanas después se extrajo la prótesis por infección de esta por Klebsiella pneumoniae. El paciente recibió radioterapia y tanto en la exploración como en la TC de control, está libre de enfermedad.

Caso 5. Varón de 80 años que consultó por lesión en paladar duro y blando derechos de un año de evolución. Se realizó una exéresis de esta con cierre simple en diciembre de 2019 y el estudio histopatológico confirmó que se trataba de un adenocarcinoma polimorfo de paladar blando y duro de bajo grado pT1 N0 Mx con positividad para CK-7, S-100, BCL2 en forma débil para SOX. CD-117 y P-63 negativas. Por el momento, el paciente se encuentra libre de enfermedad.

CAso 6. Varón de 78 años con carcinoma escamoso de paladar blando que contactaba con paladar duro izquierdo que se intervino en agosto de 2019. Se realizó una exéresis la lesión y reconstrucción con bola de Bichat más vaciamiento ganglionar de las áreas I-V izquierdas (pT1-2 N0 M0). El paciente está libre de enfermedad en el momento actual.

\section{DISCUSIÓN}

Se trata de cuatro varones y dos mujeres, de edad avanzada (media 74,5 años). El número total de pacientes con carcinomas de cabeza y cuello en 2018 y 2019 en nuestro servicio ha sido de 159 de los que aproximadamente el 10\% correspondían a tumores de orofaringe. En nuestra serie, los carcinomas de paladar blando representan $35 \%$ de los tumores de orofaringe. Cuatro de ellos son carcinomas escamosos, un carcinoma adenoide quístico y otro de ellos, un adenocarcinoma polimorfo de bajo grado. 


\section{CARCINOMA DE PALADAR BLANDO. DESCRIPCIÓN DE UNA SERIE DE CASOS \\ ZABALETA-LÓPEZ M; RAMOS-CASADEMONT L; GÓMEZ-TOMÉ E; DÍAZ DE CERIO-CANDUELA P; IBÁÑEZ-MUÑOZ C; MARTÍNEZ-TORRE M I}

La mitad de los casos son tumores localmente avanzados en el momento del diagnóstico. El seguimiento es muy breve al tratarse de casos recientes. A pesar de ello, uno de los casos ha fallecido por causas no relacionadas con su enfermedad oncológica.

Los factores predisponentes más conocidos de los tumores de orofaringe son el tabaco, el consumo de alcohol y el VPH, que cada vez adquiere más relevancia. La asociación del alcohol y el tabaco tiene un efecto sinérgico [7]. La prevalencia de infección por VPH en el carcinoma de orofaringe varía desde el 4\% al 93\%. El biomarcador más utilizado para detectar presencia de VPH es el P16 $\mathrm{y}$ tinciones $>70 \%$ se consideran positivas y muestran actividad del VPH [8]. La prevalencia media en el sur de Europa es del 17\% aunque en España es discretamente inferior [9]. Sin embargo, siendo el paladar blando una sublocalización de la orofaringe, los carcinomas suelen ser VPH negativos [3]. Se desconoce la razón por la que esto es así y algunos autores suponen que se debe a la ausencia de tejido linfoide en el paladar blando [10].

En nuestra serie, los tumores de paladar blando representaban el 35\% de los tumores de orofaringe. La mayoría de los estudios incluyen los carcinomas de paladar blando dentro de los de orofaringe o, menos frecuentemente, cavidad oral $[1,11]$. Sin embargo, algunos autores [1] postulan que los carcinomas de paladar blando tienen una etiología y patogénesis diferente a los de orofaringe, ya que, no dependen del anillo linfático de Waldeyer.

El $85 \%$ al $90 \%$ de los tumores malignos del paladar blando derivan de células epiteliales y corresponden a carcinomas escamosos. En segundo lugar, se encuentran los tumores que se originan a partir del epitelio de las glándulas salivares (en el paladar blando se localiza el 50\% de las glándulas salivares accesorias) siendo los tipos histológicos más frecuentes el carcinoma adenoide quístico y el carcinoma mucoepidermoide. Nuestros casos fueron mayoritariamente carcinomas escamosos, aunque hubo un caso de carcinoma adenoide quístico y otro adenocarcinoma polimorfo de bajo grado.

Los tumores del paladar blando son asintomáticos en fases iniciales. Cuando aumentan de tamaño pueden provocar dolor, halitosis, sangrado, rinolalia y trismus por infiltración de las estructuras vecinas como la musculatura pterigoidea [12]. Distintos autores $[1,13]$ ponen de manifiesto que los tumores de paladar blando se diagnostican en fases precoces por la facilidad diagnóstica por la localización. En nuestro caso, el 50\% eran localmente avanzados, de acuerdo con otros autores [2, 9]. Si bien su localización es fácilmente accesible a la exploración básica por parte de los médicos de atención primaria y odontoestomatólogos, inicialmente son asintomáticos y esto puede retrasar el diagnóstico de los pacientes.

La exploración endoscópica es de gran ayuda para la adecuada valoración de la extensión local del tumor y detección de segundos tumores primarios. La imagen de banda estrecha (narrow band imaging, NBI) es una herramienta muy útil en cuanto a sensibilidad y precisión, para realizar biopsias dirigidas bajo esta óptica en las lesiones del paladar [14].

En el caso de tumores localizados, la resonancia magnética nuclear (RMN) en el plano coronal con gadolinio es la prueba de elección $[15,16]$ por la mejor definición de los tejido blandos. Sin embargo, habitualmente es necesario realizar también una TC del cuelloy tórax para el estudio de extensión [15]. Casi la totalidad de nuestros pacientes (5/6) el tumor contactaba o afectaba también al paladar duro y la TC resulta necesaria [17].

Es importante saber que, aproximadamente, un $25 \%$ de los pacientes con un carcinoma de paladar blando tendrá un segundo tumor primario y la localización más habitual es el suelo de la boca [18].

El tratamiento de los tumores de paladar blando es controvertido. Hasta la última década, el tratamiento de elección ha sido la radioterapia 


\section{CARCINOMA DE PALADAR BLANDO. DESCRIPCIÓN DE UNA SERIE DE CASOS \\ ZABALETA-LÓPEZ M; RAMOS-CASADEMONT L; GÓMEZ-TOMÉ E; DÍAZ DE CERIO-CANDUELA P; IBÁÑEZ-MUÑOZ C; MARTÍNEZ-TORRE M I}

asociada a quimioterapia. En una publicación de Amy Y. Chen (2013) [5] se describe la evolución de los tratamientos del cáncer de orofaringe entre los años 1998 y 2009. Los pacientes que se trataron con quimio-radioterapia aumentaron del $22 \%$ en 1998 a $61 \%$ en 2009 . La cirugía pasó de $41 \%$ a $31 \%$ en 2009.

El avance en las nuevas técnicas quirúrgicas mediante abordaje transoral ha permitido que se aumente el número de tumores del paladar tratados quirúrgicamente. El incremento en su incidencia causado por el VPH, junto con el mejor pronóstico pese a la extensión ganglionar y la presentación en una población más joven ha impulsado el tratamiento quirúrgico.

Hasta la fecha, no hay evidencia que compare el resultado del tratamiento quirúrgico frente a la quimiorradioterapia en carcinomas de orofaringe $[19,20]$ ni tampoco estudios a largo plazo que demuestren la superioridad de la quimiorradioterapia frente a la cirugía [1].

El tratamiento quirúrgico tiene varias ventajas con respecto a la radioterapia sola o asociada a quimioterapia:
- Mejor estadificación del tumor. Sabemos que hasta en un $40 \%$ de los casos, la estadificación clínica es diferente a la quirúrgica [21].

- Se evita o se precisa una menor dosis de tratamiento complementario con radioterapia y se evita el tratamiento con quimioterapia y, por ende, las toxicidades de ambos [22-24].

- Una menor morbilidad y afectación de la deglución postoperatoria que a largo plazo es normal en la mayoría de los casos.

En los tumores del paladar blando la probabilidad de metástasis cervicales ocultas es aproximadamente del $20 \%$, por lo que está indicado el vaciamiento cervical ipsilateral de las áreas II-IV [25]. En los tumores de línea media o próximos a ella, el vaciamiento debe ser bilateral. En pacientes con evidencia radiológica de afectación de los ganglios retrofaríngeos, se debe realizar un vaciamiento de dicha área o administrar radioterapia postoperatoria.

Nuestros casos diagnosticados en estadios avanzados se trataron con quimiorradioterapia o radioterapia paliativa. Los T1-T2 se trataron quirúrgicamente, y en el caso del T3 se realizó la exéresis quirúrgica con reconstrucción y se

Tabla 1. Características de los colgajos para la reconstrucción del paladar blando [22-25].

\begin{tabular}{|c|c|c|c|c|}
\hline $\begin{array}{l}\text { Tipo de } \\
\text { colgajo }\end{array}$ & \multicolumn{2}{|l|}{ Ventajas } & Desventajas & Características \\
\hline $\begin{array}{l}\text { Bola de } \\
\text { Bichat }\end{array}$ & \multicolumn{2}{|c|}{$\begin{array}{l}\text { Rápido y sencillo. } \\
\text { Puede realizarse bajo anestesia local. Poca } \\
\text { morbilidad. } \\
\text { Puede asociarse a otros colgajos pediculados. }\end{array}$} & $\begin{array}{l}\text { Solo puede cubrir } \\
\text { pequeños o medianos } \\
\text { defectos. }\end{array}$ & Epitelización en 3-4 semanas. \\
\hline \multirow[t]{3}{*}{$\begin{array}{l}\text { Músculo } \\
\text { bucinador }\end{array}$} & $\begin{array}{l}\text { De base } \\
\text { posterior (o } \\
\text { de Bozola) }\end{array}$ & $\begin{array}{l}\text { Rápido y sencillo. } \\
\text { Cierre primario del defecto. } \\
\text { La radiación previa no se } \\
\text { considera contraindicación. }\end{array}$ & Puede producir trismus. & $\begin{array}{l}\text { Arteria a bucal o facial. } \\
\text { Reconstrucción en pacientes } \\
\text { edéntulos. } \\
\text { También, reconstrucción de } \\
\text { pared faríngea lateral. }\end{array}$ \\
\hline & FAMM & $\begin{array}{l}\text { Rápido y sencillo. } \\
\text { Cierre primario del defecto. }\end{array}$ & $\begin{array}{l}\text { Defectos pequeños. } \\
\text { No se puede realizar si } \\
\mathrm{N}+\end{array}$ & $\begin{array}{l}\text { Se puede pedicular superior } \\
\text { o anteriormente. Defectos de } \\
\text { hasta } 2 \mathrm{~cm} \text {. }\end{array}$ \\
\hline & De Zhao & $\begin{array}{l}\text { Se puede utilizar en pacientes no } \\
\text { edéntulos. } \\
\text { No se necesita una sección del } \\
\text { pedículo en un segundo tiempo }\end{array}$ & \multicolumn{2}{|c|}{ Limitado por la comisura labial. No se puede realizar si $\mathrm{N}_{+}$} \\
\hline
\end{tabular}




\section{CARCINOMA DE PALADAR BLANDO. DESCRIPCIÓN DE UNA SERIE DE CASOS \\ ZABALETA-LÓPEZ M; RAMOS-CASADEMONT L; GÓMEZ-TOMÉ E; DÍAZ DE CERIO-CANDUELA P; IBÁÑEZ-MUÑOZ C; MARTÍNEZ-TORRE M I}

administró radioterapia postoperatoria. En todos ellos se realizó un vaciamiento cervical ganglionar unilateral o bilateral.

La resección de los tumores del paladar blando puede tener como secuela una insuficiencia velopalatina, fundamentalmente por la sección del elevador del velo del paladar y también por una disfunción de la trompa de Eustaquio (por una lesión del tensor del velo del paladar). En éstos casos está indicada una reconstrucción cuyos objetivos deben [26]:

- Mantener la separación de la orofaringe y nasofaringe.

- Conservar la permeabilidad nasal.

- Respetar la inteligibilidad y resonancia del habla adecuadas.

- Asegurar una deglución correcta.

- Permitir la vigilancia del tumor y ser coste efectiva.

La mayoría de los autores cree que la reconstrucción funcional del paladar está indicada en defectos que representen más del 50\% (Tabla 1). Para defectos pequeños $(<50 \%)$, existen como opciones la bola de Bichat o el colgajo de músculo buccinador. El primero va a cubrir defectos pequeños o medianos de forma rápida y sencilla, confiriendo poca morbilidad a la cirugía y epitelizándose en 3 a 4 semanas. El segundo tiene diferentes variantes como el colgajo de base posterior (o de Bozola) o el colgajo miomucoso de arteria facial (FAMM) (Figura 3). Ambos son rápidos y sencillos de realizar, permitiendo un cierre primario del defecto de la zona donante. En el caso del FAMM se debe de tener en cuenta, si es necesario un vaciamiento ganglionar, la preservación de la arteria facial [27].

Para aquellos defectos mayores del 50\% también se dispone de distintas opciones como son el colgajo miomucoso infrahioideo, el colgajo de músculo temporal, el colgajo en isla de la arteria facial tunelizada, el colgajo en isla junto con el colgajo de pared faríngea lateral y el colgajo microvascular de arteria radial [28]. El colgajo de músculo temporal es fácil, seguro y rápido de realizar, aunque puede llegar a ser bastante voluminoso. El colgajo microvascular de arteria radial tiene la ventaja de poder combinarse con otros tipos de colgajos para reconstruir defectos mayores o para la reconstrucción tras la radiación [29], pero requiere experiencia con la microcirugía. Finalmente, los dos colgajos en isla están indicados cuando se precisa de una reconstrucción total del paladar [30].

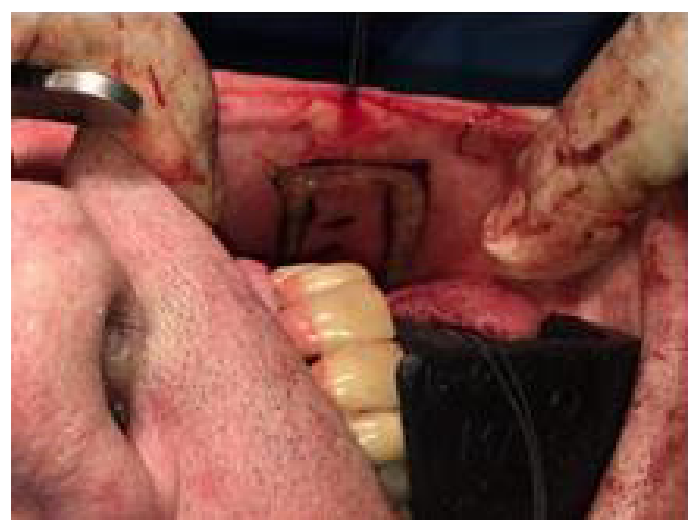

Figura 3 Diseño del colgajo miomucoso de arteria facial, FAMM.

\section{CONCLUSIONES}

Los carcinomas del paladar blando son poco sintomáticos en fases iniciales, por lo que se requiere un alto índice de sospecha para evitar que sean diagnosticados en fases avanzadas. Los médicos de atención primaria y los odontoestomatólogos son piezas claves en la sospecha diagnóstica de estos tumores.

El tratamiento óptimo de estos tumores no está bien definido, si bien, el desarrollo de la cirugía transoral, con una menor morbilidad, ha dado un impulso al tratamiento quirúrgico.

El tratamiento quirúrgico del cáncer de paladar mediante los abordajes transorales permiten reducir 


\section{CARCINOMA DE PALADAR BLANDO. DESCRIPCIÓN DE UNA SERIE DE CASOS \\ ZABALETA-LÓPEZ M; RAMOS-CASADEMONT L; GÓMEZ-TOMÉ E; DÍAZ DE CERIO-CANDUELA P; IBÁÑEZ-MUÑOZ C; MARTÍNEZ-TORRE M I}

la necesidad de tratamiento complementario hasta en un $40 \%$ de los casos.

La reconstrucción se hace necesaria en resecciones mayores del 50\%.

\section{BIBLIOGRAFÍA}

1. Iyer NG, Nixon IJ, Palmer F, Kim L, Whitcher M, Katabi N, et al. Surgical management of squamous cell carcinoma of the soft palate: Factors predictive of outcome. Head Neck. 2012;34(8):107180. Disponible en: http://doi.wiley.com/10.1002/ hed.21878 [Citado el 21/6/2020]

2. Chow LQM. Head and Neck Cancer. Longo DL, editor. NEngl J Med[Internet]. 20202;382(1):60-72. Disponible en: http://www.nejm.org/doi/10.1056/ NEJMra1715715 [Citado el 23/6/2020]

3. Jouhi L, Atula T, Mäkitie A, Keski-Säntti H. Management of clinically N0 neck in oropharyngeal carcinoma. Eur Arch Oto-RhinoLaryngology. 2019 7;276(4):1205-10. Disponible en: http://link. springer.com/10.1007/s00405-01905314-x [Citado el 24/6/2020]

4. Douglas WG, Rigual NR, Giese W, Bauer J, Wiseman SM, Loree TR, et al. Advanced soft palate cancer: The clinical importance of the parapharyngeal space. Otolaryngol Head Neck Surg. 2005;133(1):66-9. [Citado el 21/6/2020]

5. Chen AY, Zhu J, Fedewa S. Temporal Trends in Oropharyngeal Cancer Treatment and Survival : 1998-2009. Laryngoscope. 2014;124:131-8. [Citado el 22/6/2020]

6. Ángel J, García G, Pollán C, Virós D, Miguel C, Estomba C, et al. Reconstrucción de defectos orofaríngeos tras cirugía transoral robótica . Revisión y recomendaciones de la Comisión de Cirugía de Cabeza y Cuello de la Sociedad Española de Otorrinolaringología y Cirugía de Cabeza y Cuello. 2018;1-10. [Citado el 22/6/2020]

7. Jethwa AR, Khariwala SS. Tobacco-related carcinogenesis in head and neck cancer. Cancer Metastasis Rev. 2017 12;36(3):411-23. Disponible en: http:// link.springer.com/10.1007/s10555-0179689-6 [Citado el 23/6/2020]

8. Frandsen VL, Grønhøj Larsen C, von Buchwald C. Prevalence of human papillomavirus in squa- mous cell carcinomas of the soft palate: Table 1. J Clin Pathol. 2015; 68(11):942-3. Disponible en: http://jcp.bmj.com/lookup/doi/10.1136/jclinpath2015-203081 [Citado el 25/6/2020]

9. Rodrigo JP, Heideman DAM, García-Pedrero JM, Fresno MF, Brakenhoff RH, Díaz Molina JP, et al. Time trends in the prevalence of HPV in oropharyngeal squamous cell carcinomas in northern Spain (1990-2009). Int J Cancer. 2014;134(2):487-92. [Citado el 23/6/2020]

10. Rapp CT, Chera BS, Morris CG, Amdur RJ, Kirwan JM, Mendenhall WM. Radiation treatment of soft palate squamous cell carcinoma. Vol. 42, Head \& Neck. 2020. p. 530-8. Disponible en: https://onlinelibrary.wiley.com/ doi/abs/10.1002/h ed.26035 [Citado el 24/6/2020]

11. Schernberg A, Canova C, Blanchard P, Gorphe P, Breuskin I, Mirghani H, et al. Prognostic factors in patients with soft palate squamous cell carcinoma. Head Neck. 2019 12;41(5):1441-9. Disponible en: https://onlinelibrary.wiley.com/doi/ abs/10.1002/h ed.25598 [Citado el 23/6/2020]

12. Basterra Alegría J. Carcinomas de la faringe. Ponencia oficial del LVIII Congreso Nacional de la Sociedad de Otorrinolaringología y Patología Cervicofacial. 2007. [Citado el 25/6/2020]

13. Chan CK, Han AY, Alonso JE, Xu MJ, Mallen-St Clair J, Heaton CM, et al. Squamous Cell Carcinomaofthe Soft Palate in the United States: A Population-Based Study. Otolaryngol Neck Surg. 2018 8;159(4):662-8. Disponible en: https://doi.org/10 $.1177 / 0194599818771931$ [Cita do el 23/6/2020]

14. Piazza C, Del Bon F, Paderno A, Grazioli P, Perotti $\mathrm{P}, \mathrm{Barbieri} \mathrm{D}$, et al. The diagnostic value of narrow band imaging in different oral and oropharyngeal subsites. Eur Arch Oto-RhinoLaryngology. 2016 15;273(10):3347-53. Disponible en: http://link. springer.com/10.1007/s00405-0163925-5 [Citado el 23/6/2020]

15. Mehanna H, Evans M, Beasley M, Chatterjee S, Dilkes M, Homer J, et al. Oropharyngeal cancer: United Kingdom National Multidisciplinary Guidelines. J Laryngol Otol. 2016;130(S2):S90-6. [Citado el 24/6/2020]

16. Lewis-Jones H, Colley S, Gibson D. Imaging in head and neck cancer: United Kingdom National 


\section{CARCINOMA DE PALADAR BLANDO. DESCRIPCIÓN DE UNA SERIE DE CASOS \\ ZABALETA-LÓPEZ M; RAMOS-CASADEMONT L; GÓMEZ-TOMÉ E; DÍAZ DE CERIO-CANDUELA P; IBÁÑEZ-MUÑOZ C; MARTÍNEZ-TORRE M I}

Multidisciplinary Guidelines. J Laryngol Otol. 2016;130(S2):S28-31. [Citado el 22/6/2020]

17. Kato H, Kanematsu M, Makita H, Kato K, Hatakeyama D, Shibata T, et al. CT and MR imaging findings of palatal tumors. Eur J Radiol. 2014;83(3):e137-46. Disponible en: https:// linkinghub.elsevier.com/retrieve/pii/S0720 048X13006426 [Citado el 25/6/2020]

18. Osborne RF, Brown JJ. Carcinoma of the oral pharynx: an analysis of subsite treatment heterogeneity. Surg Oncol Clin N Am. 2004;13(1):7180. Disponible en: https://linkinghub.elsevier. com/retrieve/pii/S1055 320703001170 [Citado el 25/6/2020]

19. Beitler JJ, Quon H, Jones CU, Salama JK, Busse PM, Cooper JS, et al. ACR Appropriateness Criteria ${ }^{\circledR}$ Locoregional therapy for resectable oropharyngeal squamous cell carcinomas. Head Neck. 2016;38(9):1299-309. [Citado el 24/6/2020]

20. Head and neck cancers. National Comprehensive Cancer Network. NCCN Guidelines Version 2.2020. Head and Neck Cancers. 2020. [Citado el 27/6/2020]

21. Shah S, Goldenberg D. Robotic surgery for oropharyngeal cancer. Rambam Maimonides Med J. 2014;5(2):e0014. [Citado el 25/6/2020]

22. Genden EM, Kotz T, Tong CCL, Smith C, Sikora AG, Teng MS, et al. Transoral Robotic Resection and Reconstruction for Head and Neck Cancer. Laryngoscope. 2011;12:1668-74. [Citado el 24/6/2020]

23. Adelstein DJ, Ridge JA, Brizel DM, Holsinger FC, Haughey BH, O'Sullivan B, et al. Transoral resection of pharyngeal cancer: Summary of a National Cancer Institute Head and Neck Cancer Steering Committee Clinical Trials Planning Meeting, November 6-7, 2011, Arlington, Virginia. Head Neck. 2012;34(12):1681-703. [Citado el 24/6/2020]
24. López F, Llorente JL, Álvarez-Marcos C, Morato M, Suárez C, Rodrigo JP. Resultados del tratamiento de los carcinomas epidermoides orofaríngeos mediante cirugía transoral. Acta Otorrinolaringol Esp. 2015;66(2):74-82. [Citado el 23/6/2020]

25. Fossum CC, Chintakuntlawar A V, Price L, Garcia JJ. Characterization of the Oropharynx: Anatomy, Histology, Immunology, Squamous Cell Carcinoma \& Surgical Resection. Histopathology. 2016;70(7):1021-9. [Citado el 22/6/2020]

26. Seikaly H, Rieger J, Zalmanowitz J, Tang JL, Alkahtani K, Ansari K, et al. Functional soft palate reconstruction: a comprehensive surgical approach. Head Neck. 2008;1615-23. [Citado el 24/6/2020]

27. Bianchi B, Ferri A, Ferrari S, Copelli C. Myomucosal cheek flaps: applications in intraoral reconstruction. Oral Surg Oral Med Oral Pathol Oral Radiol Endod. 2009;108(3):353-9. [Citado el 26/6/2020]

28. Zabaleta-López M, Ramos-Casademont L, Díaz de Cerio-Canduela P, Ibáñez-Muñoz C, LacostaNicolás JL, Carreras-Alcaraz A. Anatomía quirúrgica en el carcinoma de paladar blando. Revisión bibliográfica. Revista ORL. Disponible en: https:// revistas.usal.es/index.php/24447986/article/ view/23103. DOI: http://dx.doi.org/10.14201/ orl.23103. [Citado el 30/6/2020]

29. Moubayed SP, Osorio M, Buchbinder D, Lazarus C, Urken ML. Soft palate reconstruction using a combination of a turn-in flap and a radial forearm flap. Laryngoscope. 2017;127(8):1772-4. [Citado el 24/6/2020]

30. Karle WE, Anand SM, Clain JB, Scherl S, Urken ML. Total Soft Palate Reconstruction Using the Palatal Island and Lateral Pharyngeal Wall Flaps. Laryngoscope. 2013:929-33. [Citado el 25/6/2020] 